\title{
Anaerobic microbiota facilitate $P$. aeruginosa access to the airway epithelium in a novel co-culture model of colonization
}

\section{Patrick J. Moore ${ }^{1}$, Talia D. Wiggen ${ }^{1}$, Leslie A. Kent ${ }^{1}$, Sabrina J. Arif ${ }^{1}$, Sarah K. Lucas ${ }^{1}$, Scott M.} O'Grady², Ryan C. Hunter ${ }^{1 \#}$

${ }^{1}$ Department of Microbiology \& Immunology, University of Minnesota, $68923^{\text {rd }}$ Avenue SE, Minneapolis, MN 55455

${ }^{2}$ Department of Animal Science, University of Minnesota, 1364 Eckles Avenue, Saint Paul, MN 55108

\#To whom correspondence should be addressed:

Ryan C. Hunter

Department of Microbiology \& Immunology

Microbiology Research Facility, 3-115

University of Minnesota

$68923^{\text {rd }}$ Avenue SE

Minneapolis, MN 55455

Tel: (612) 625-1402

Email: rchunter@umn.edu

\section{ABSTRACT}

The role(s) of anaerobic microbiota in chronic airway disease are poorly understood due to inherent limitations of existing laboratory models. To address this knowledge gap, we use a dual oxic-anoxic coculture approach that maintains an oxygen-limited apical epithelial microenvironment while host cells are oxygenated basolaterally. Reduced oxygen culture did not alter the physiology or gene expression of Calu3 cells but supported anaerobe-epithelial interactions for $24 \mathrm{~h}$ without affecting bacterial or host cell viability. Anaerobe challenge led to increased expression of inflammatory marker genes and compromised integrity of apical mucins, leading to our hypothesis that anaerobe-host interactions prime the airways for chronic infection. Indeed, anaerobe pre-treatment of Calu-3 cells led to an increase in Pseudomonas aeruginosa colonization. This model system offers new insight into anaerobe-host interactions in airway disease pathophysiology and motivates further study of the lung, gut, and oral cavity, where etiological roles of anaerobes have been proposed but specific pathogenic mechanisms remain unclear. 


\section{INTRODUCTION}

Decades of clinical lab culture have focused on a limited set of pathogens associated with acute and chronic airway disease (e.g. Pseudomonas aeruginosa, Staphylococcus aureus, Mycobacterium tuberculosis). More recently, culture-independent studies of airway microbiota have identified more complex bacterial signatures, lending evidence to polymicrobial disease etiologies. Notably, oral and supraglottic-associated facultative and obligate anaerobes - Prevotella, Veillonella, Streptococcus spp. are present at low densities in the healthy respiratory tract (1-4) and are both prevalent and abundant in chronic obstructive pulmonary disease (COPD) $(5,6)$, cystic fibrosis (CF) (7), non-CF bronchiectasis (8), lung abscess (9), sinusitis (10), idiopathic pulmonary fibrosis (11), and tuberculosis (12). While salivary contamination during sampling remains controversial, consensus is that the development of hypoxic microenvironments within diseased airway mucus provides a niche for anaerobe proliferation, often reaching densities equal to or greater than those of canonical pathogens $(7,13)$.

The function of anaerobic microbiota in airway disease is poorly understood though several roles have been proposed. In healthy individuals, anaerobe abundance in bronchoalveolar lavage fluid correlates with expression of proinflammatory cytokines, elevated Th17 lymphocytes, and a blunted TLR4 response, implicating a compromised first line of defense against bacterial infection (1). Indeed, epidemiologic and in vitro data suggest that anaerobes may facilitate secondary colonization by canonical airway pathogens. In non-CF bronchiectasis, Prevotella and Veillonella positively correlate with Th17 cytokines and non-tuberculosis mycobacterial infection (14). Similarly, in HIV subjects, anaerobes suppress expression of interferon gamma and IL-17A via production of short-chain fatty acids (SCFAs) and are thought to impair the host response to consequent M. tuberculosis colonization (12). In CF, anaerobe-derived SCFAs increase with age and disease progression (15), mediate excessive production of IL-8 by bronchial epithelial cells (in turn promoting neutrophil mobilization) (16) and potentiate the growth and virulence of canonical CF pathogens in vitro $(17,18)$. CF anaerobes have also been reported to increase in abundance during pulmonary exacerbations prior to antibiotic therapy (19), further implicating their role in pathogenesis. 
While these data collectively support the causality of anaerobic microbiota in airway disease, direct mechanistic studies of anaerobe-host and anaerobe-host-pathogen interactions have been limited by the paucity of compatible laboratory methods. Animal models poorly reflect chronic infection pathologies and are prohibitively expensive for high throughput analyses. As an alternative, the development of threedimensional (3D) cell cultures have greatly expanded our knowledge of host-microbe interactions at the respiratory epithelial interface (20-22). However, incorporation of anaerobic microbiota into these models is restricted by the inherent challenge of maintaining host cell viability under hypoxic or anoxic culture conditions. New models are needed for a deeper understanding of the role of anaerobic microbiota in acute and chronic airway disease.

A novel system was recently described that enables co-culture of human intestinal enteroids with obligately anaerobic microbiota under reduced oxygen conditions that recapitulate hypoxic microenvironments found in vivo (23). Here, we adopted this approach to overcome limitations of studying anaerobe interactions with the airway epithelium in 3D cell culture. First, polarized monolayers of the mucus-overproducing epithelial cell-line, Calu-3, were maintained at air-liquid interface in an anaerobic chamber while $\mathrm{O}_{2}$ and $\mathrm{CO}_{2}$ were delivered through a chamber entry port. This setup allows for maintenance of an anoxic microenvironment in the apical compartment while host cells are oxygenated basolaterally. Using this culture system, we establish its utility for the in vitro study of anaerobe-airway interactions. We then use this model to test the hypothesis that anaerobic microbiota enhance colonization of the epithelial surface by $P$. aeruginosa. Data presented here not only demonstrate the power and versatility of the anoxic co-culture approach, but also offer new insight into the mechanisms of pathogen colonization and a potential role of anaerobic microbiota in the development of airway infection.

\section{RESULTS}

Optimization and validation of a dual oxic-anoxic airway epithelial culture system. The primary objective was to establish a cell culture system that facilitates study of anaerobe-host interactions (Figure 1). We first cultured polarized monolayers of the adenocarcinoma cell line, Calu-3, at air-liquid interface (ALI) for 21-28 days under standard (normoxic) conditions. As shown previously (24), polarized Calu-3s produce a distinct mucus layer on the apical surface (Figure S1), mimicking aberrant mucin accumulation 
associated with chronic airway disease. Once polarized, cell cultures were placed in a gas-permeable multi-well plate manifold (Figure S2), transferred to anaerobic chamber, and mixed gas $\left(21 \% \mathrm{O}_{2} / 5 \% \mathrm{CO}_{2} /\right.$ $74 \% \mathrm{~N}_{2}$ ) was delivered through a chamber port to the basolateral compartment of the Transwell-containing plate. Cells were cultured for an additional $24 \mathrm{~h}$ at anoxic liquid interface (ANLI) prior to analysis.

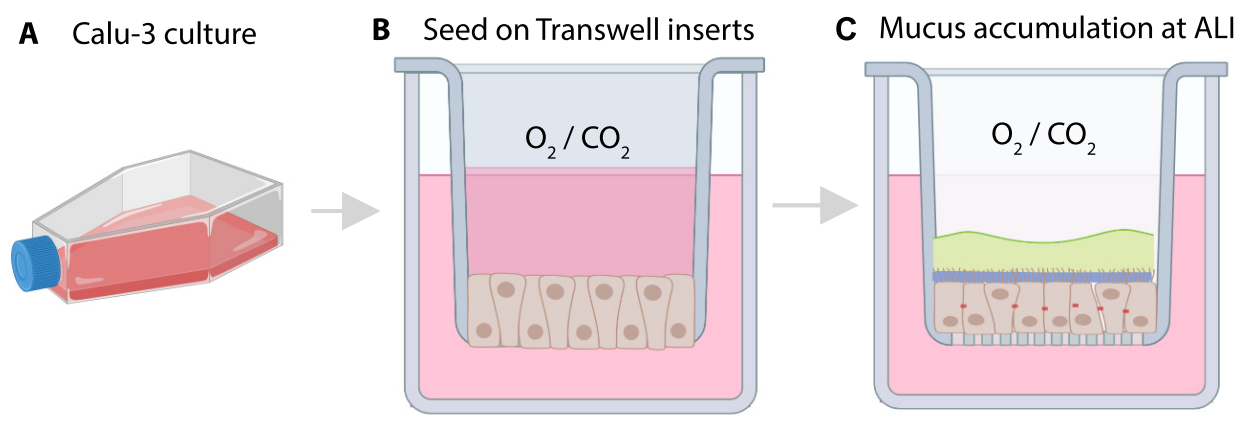

Figure 1. Calu-3 culture at anoxic liquid interface (ANLI). (A) Calu-3 cells are cultured in MEM with $10 \%$ FBS. (B) Cells are seeded on $6.5 \mathrm{~mm}$ Transwell inserts and grown to confluency ( $\sim 5$ days). (C) Apical medium is removed, and cells are cultured at air-liquid interface (ALI) for 21-28 days prior to (D) incubation under anoxic liquid interface (ANLI) conditions, where the apical compartment is oxygen limited and mixed gas is delivered basolaterally. Figure created with BioRender.com.

We then determined the effects of ANLI culture, if any, on Calu-3 cell physiology. Quantification of lactate dehydrogenase release showed a negligible increase between normoxic and ANLI culture conditions $(p=0.42)$, suggesting little to no change in viability after $24 \mathrm{~h}$ (Figure $2 A)$. Transepithelial electrical resistance (TEER) (Figure 2B) and E-cadherin concentrations (Figure 2C), both proxies of epithelial barrier integrity, were also similar between culture conditions ( $p=0.38$ and 0.55 , respectively). These data were further supported by immunofluorescence microscopy which revealed confluent monolayers and welldefined staining of the tight junction zonula occludens protein which appeared as near-continuous rings localized to the periphery of each cell (Figure 2D).

Previous work has shown hypoxia-induced expression of pro-inflammatory cytokines in primary pulmonary fibroblasts (25). Thus, we used enzyme-linked immunosorbent assays to quantify IL-6 and IL- 

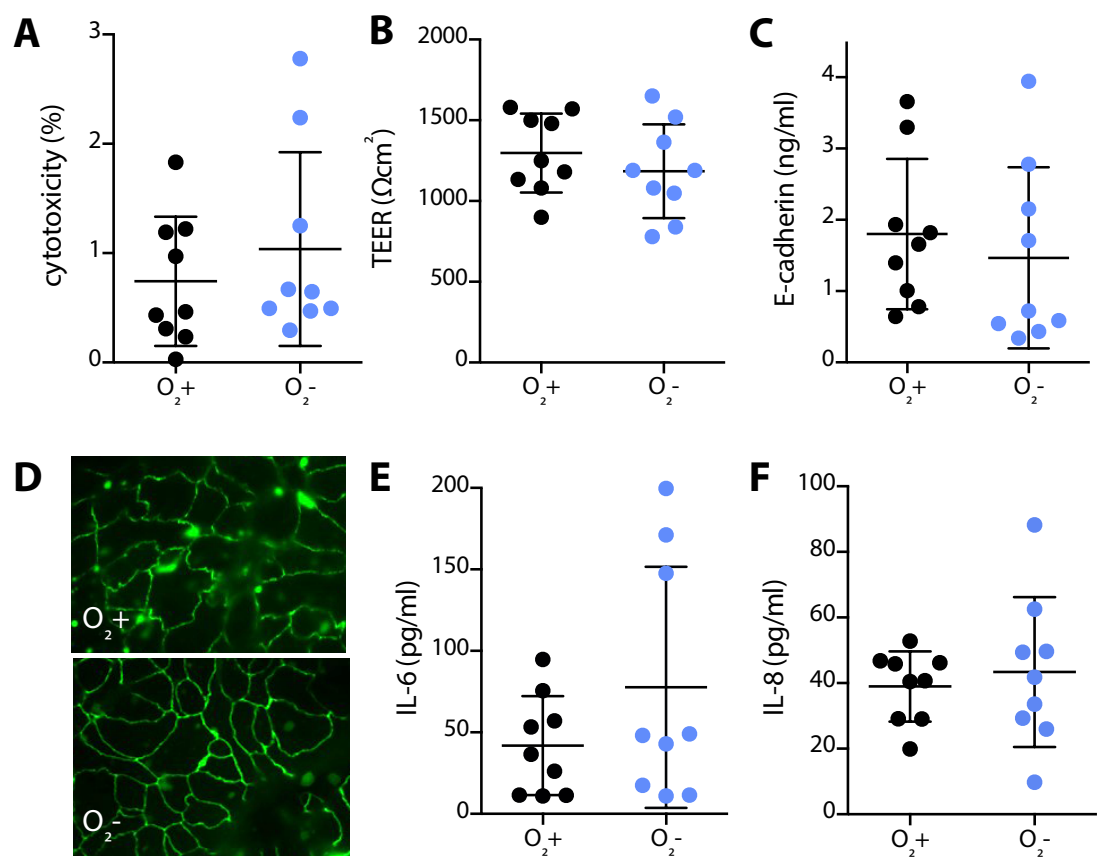

Figure 2. Culture of human bronchial epithelial cells (Calu-3) at ANLI has minimal effect on cell physiology. (A) Cytotoxicity as measured by lactate dehydrogenase (LDH) in the culture medium, (B) transepithelial electrical resistance (TEER), (C) E-cadherin concentrations, (D) immunofluorescence of zonula occludens, and cytokines (E) IL-6, and (F) IL-8 showed no significant differences between normoxic and ANLI culture conditions. All data shown are for three independent experiments using three biological replicates $(n=9)$ and were compared using an unpaired t-test with Welch's correction.

To gain a broader understanding of the physiological response of Calu-3 cells to ANLI culture, we used RNAseq to compare global Calu-3 gene expression to culture under normoxic conditions. Transcriptome analysis revealed 148 differentially expressed transcripts (117 upregulated, 31 downregulated, I2fc $\geq 1$, padj $<0.001$; out of $\sim 16,000$ total genes) (Figure 3A, Table S1). With the exception of ANGPTL4 (encoding angiopoietin-like 4) and SERPINA1 (alpha-1 antitrypsin)(Figure 3B) which are induced in response to hypoxia and acute inflammation, respectively, few markers of cell stress were differentially expressed, including genes involved in tight junction formation, oxidative stress, and endoplasmic reticulum stress. Importantly, HIF-1 $1 \alpha$, which is constitutively expressed at low levels under 
normoxia but upregulated under hypoxia, was also consistent between culture conditions after $24 \mathrm{~h}$, suggesting Calu-3 cells were sufficiently oxygenated (Figure 3B). Among inflammatory biomarkers, only ICAM1 (intracellular adhesion molecule 1) and TGF $\beta 1$ (transforming growth factor beta 1) showed significant differences, further demonstrating that ANLI culture did not yield an appreciably proinflammatory microenvironment (Figure $3 \mathrm{C}$ ). Finally, since we use this model to assay bacterial colonization of the mucus layer, we compared mucin-related gene expression between conditions. Among detectable transcripts (MUC1, MUC3A, MUC5AC, MUC5B, and MUC13), no significant differences were observed (Figure 3D). These data demonstrate that culture of a respiratory epithelial cell line at anoxic liquid interface yields minimal changes in host cell physiology.
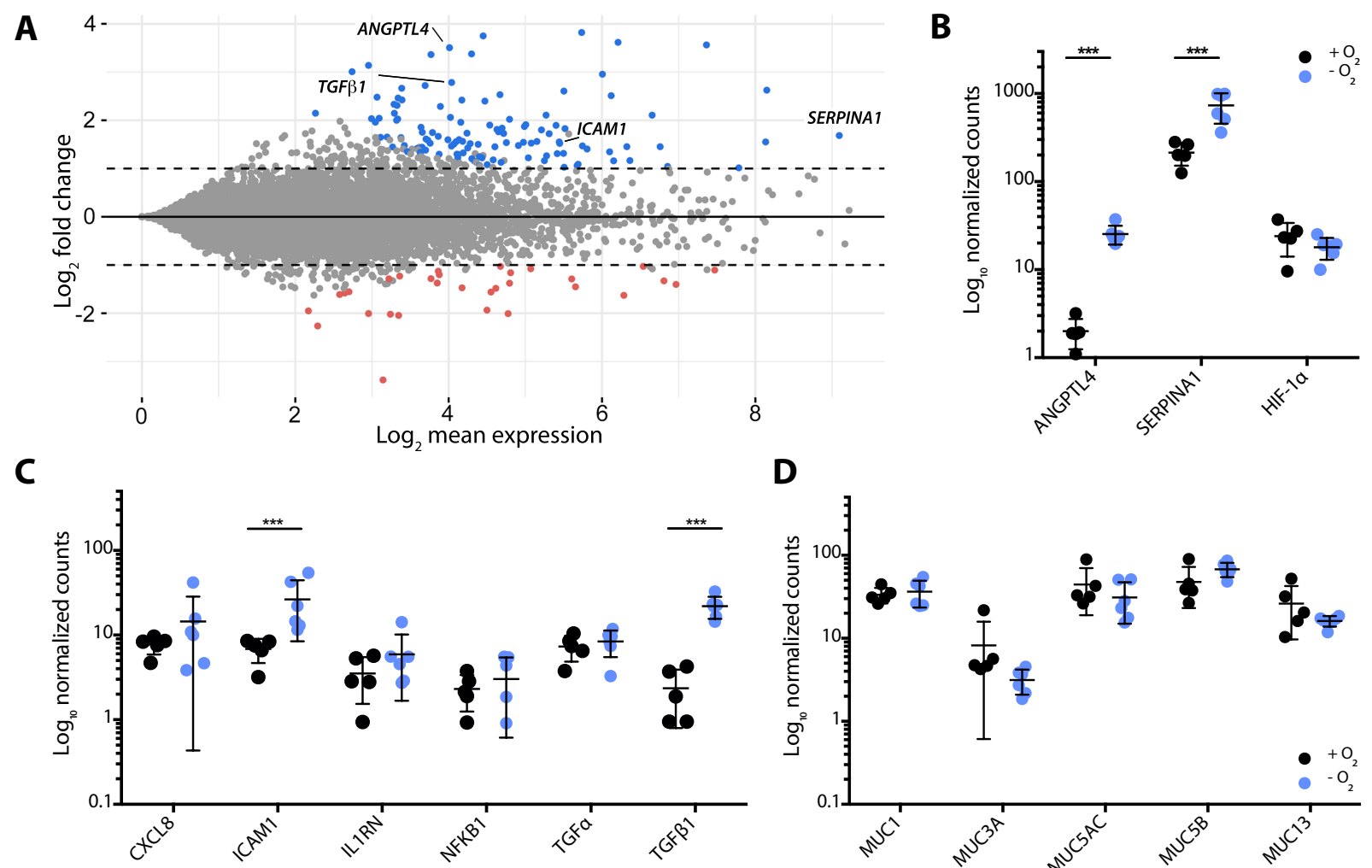

Figure 3. Culture of Calu-3 cells at ANLI yields a similar transcriptomic profile to normoxic culture conditions (ALI). (A) MA plot representation of Calu-3 gene expression at ANLI relative to normoxic culture (ALI). (B) ANGPTL4 and SERPINA1 were differentially expressed, though HIF-1 $\alpha$ was consistent between cultures. Few changes in (C) inflammatory biomarkers and (D) mucin gene expression were observed. Data shown in panels B-D are $\log _{10}$-normalized gene counts from five or six independent biological replicates and were compared using the Wald test, Benjamini-Hochberg adjusted $\left({ }^{* * *}, p<0.001\right)$. 
Anaerobic airway microbiota induce an inflammatory host response. To date, the lack of tractable cell culture systems compatible with hypoxic or anoxic growth has limited our understanding of hostanaerobe interactions. Prior work has shown that culture supernatants of anaerobic bacteria elicit proinflammatory cytokine expression in vitro through mixed-acid fermentation and production of SCFAs (1, 15, 16). However, it is not yet known how the host responds to the physical presence of anaerobes at the airway epithelial interface. To address this knowledge gap, we used our ANLI culture approach to assess the response of Calu-3 cells to co-culture with anaerobic microbiota.

As a starting point, we used a defined anaerobic bacterial consortium (ABC) enriched from airway mucus derived from an individual with chronic sinusitis. This representative community was chosen for its dominant bacterial taxa (Veillonella, Prevotella, Streptococcus) associated with both healthy and diseased airways (Figure 4A). These genera are also known for their mucin-degradation capacity and the ability to support pathogen growth through nutrient cross-feeding (17). After $3 \mathrm{~h}$ of equilibration at ANLI, Calu-3 cells were apically challenged with the anaerobic consortium ( $\left.8 \times 10^{6} \mathrm{CFUs}\right)$ and incubated for an additional 24h. Colonization was confirmed using scanning electron microscopy which revealed bacterial cells at the epithelial interface (Figure 4B). Importantly, anaerobes ( $4 \times 10^{6} \mathrm{CFUs}$ ) were recovered after $24 \mathrm{~h}$ by washing with PBS and plating on Brain Heart Infusion agar $(\mathrm{BHI})$, while washing with Triton $\mathrm{X}-100$ resulted in a $0.7-\log$ increase in recovery $\left(1.6 \times 10^{7} \mathrm{CFUs}\right)$, suggesting both anaerobe growth at the epithelial surface and either robust attachment or bacterial invasion of host cells (i.e., cells were not removed by PBS washing alone). Recovery of $\sim 7 \times 10^{5} \mathrm{CFUs}$ on a Prevotella-selective medium (Brucella Blood Agar, BBA) confirms that apical oxygen concentrations were sufficiently reduced to facilitate strict anaerobe growth. Despite this growth, cytotoxicity was not induced by anaerobe challenge after 24h (Figure 4D).

We then used RNAseq to profile the Calu-3 transcriptional response to anaerobe $(A B C)$ challenge (Figure 4E, Table S2). Contrary to our expectation, only five genes were differentially expressed relative to untreated ANLI cell cultures (all upregulated, $\mid 2 \mathrm{fc} \geq 1$, padj $<0.001$ ), though all were markers of inflammation. These included ICAM1, TNFAIP2 (mediated by TNF $\alpha$ in response to bacterial challenge) (26), chemokines CXCL1 and CXCL5 (neutrophil chemoattractants primarily expressed as an acute 

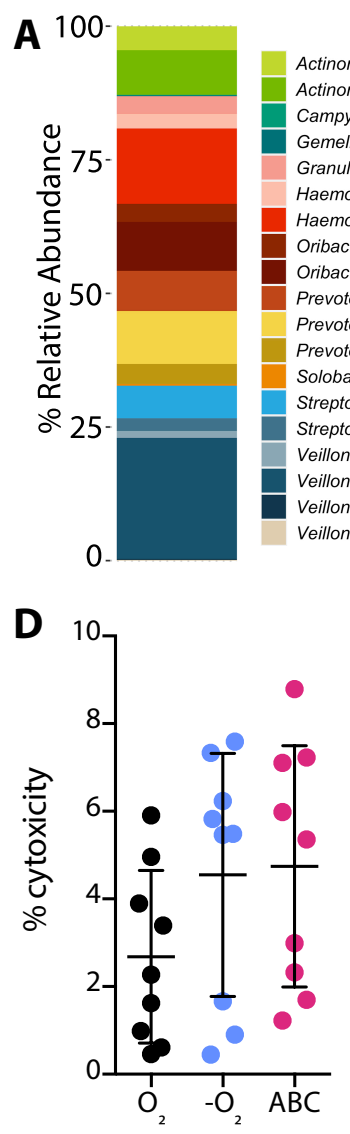

B

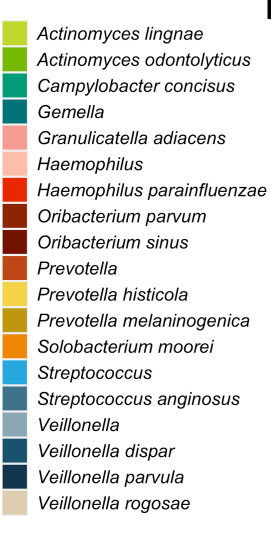

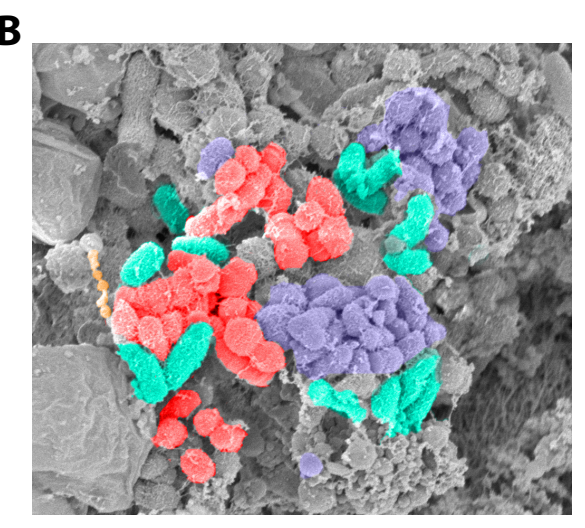

$\mathbf{E}$

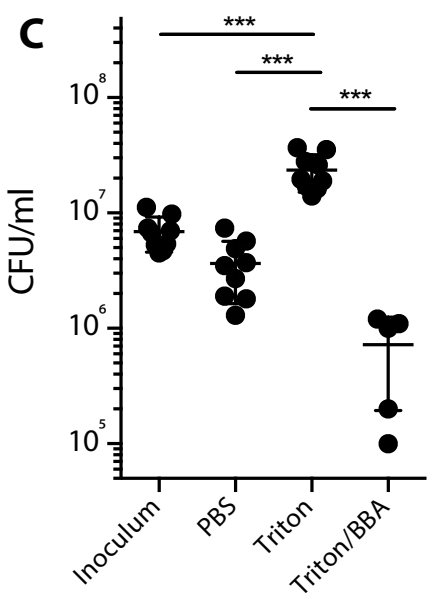

$\mathbf{F}$
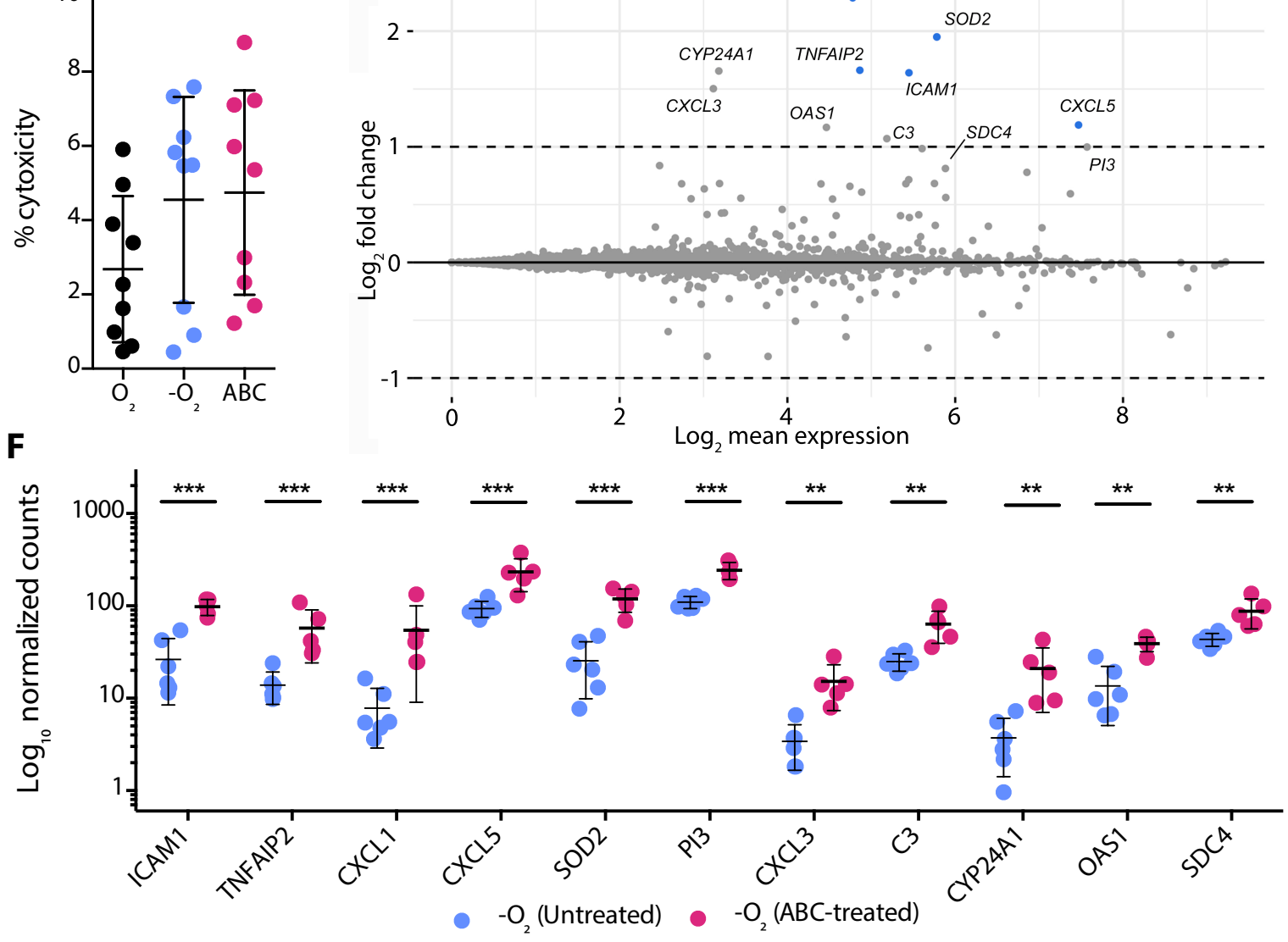

Figure 4. Anaerobic microbiota colonize the apical surface of Calu-3 cells and induce a pro-inflammatory response. (A) Taxonomic composition of an anaerobic bacterial consortium $(A B C)$ derived from the upper airways. (B) SEM micrograph of Calu-3 cells after CRS challenge. (C) Bacterial recovery from Calu-3 cells after $24 \mathrm{~h}$ by washing with PBS, TritonX-100, and plating on Prevotella selective agar (BBA). (D) Anaerobe (ABC) challenge did not result in Calu-3 cytotoxicity relative to unchallenged cells. (E) MA plot representation of Calu-3 gene expression under ANLI after ABC challenge relative to an untreated ANLI control. (F) Log $_{10}$-normalized gene counts from five or six independent biological replicates. Data in panels $C$ and $D$ were compared using a one-way ANOVA ( $p<0.0001)$ with multiple comparisons test. Data in panels $E$ and $F$ were compared using a Wald test, Benjamini-Hochberg adjusted $\left({ }^{* * *} p<0.001,{ }^{* *}<0.01\right)$. 
inflammatory response to infection) $(27,28)$, and SOD2 (superoxide dismutase 2 , expressed in response to bacterial LPS and has an antiapoptotic role against inflammatory cytokines) (29). Other inflammatory markers including PI3 (elafin, an elastase inhibitor that can prime innate immune responses in the lung) (30), CXCL3, C3 (complement), CYP24A1 (cytochrome p450 family 24 subfamily A member 1), OAS1 (oligoadenylate synthetase), and SDC4 (syndecan 4) were also differentially expressed, though did not reach our threshold padj of $<0.001$ (Figure 4F).

Anaerobic microbiota alter the mucosal interface through mucin degradation. Our previous work demonstrated the functional capacity of anaerobic microbiota to degrade airway mucins and support the growth of canonical pathogens via nutrient cross-feeding (17). Thus, in support of downstream pathogen colonization experiments, we used fast protein liquid chromatography (FPLC) to determine whether anaerobe challenge altered Calu-3 mucin integrity relative to unchallenged cells. To do so, we collected and purified mucin from the apical side of the Transwells as previously described (24) and used CL-4B size-exclusion chromatography to assay their integrity. As expected, chromatograms revealed two characteristic peaks; (i) high molecular weight mucins which ran in the void volume of the column, and (ii) a broader inclusion volume peak representative of lower molecular weight mucins $(31,32)$ (Figure 5A). While differences in the chromatographic profile of peak 1 (high molecular weight mucins) were negligible between culture conditions ( $p=0.38)$, peak 2 area was significantly reduced $(p=.007)$ following anaerobe challenge (Figure 5B), reflecting degradation of lower-molecular weight mucin glycoproteins. Together, these data suggest that in addition to eliciting a pro-inflammatory host response, anaerobic colonization alters the physicochemical properties of mucosal interface. 

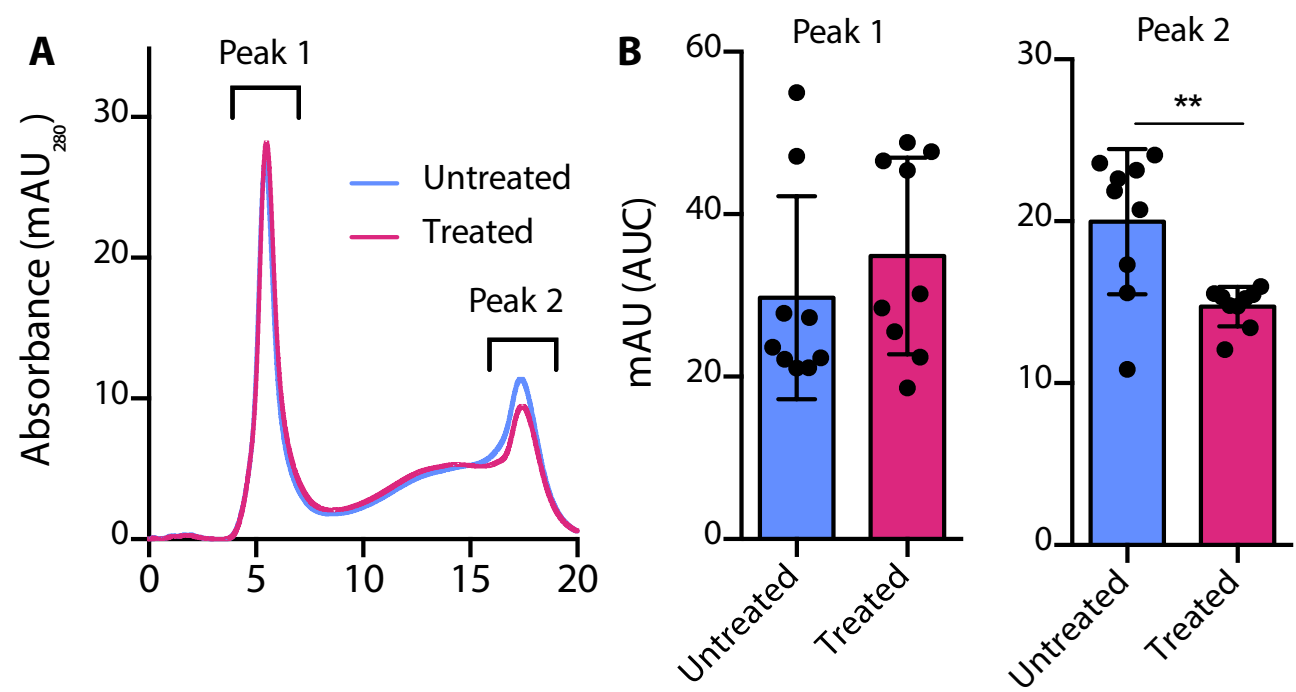

Figure 5. Anaerobic microbiota alter epithelial mucin integrity. (A) Representative FPLC traces of MUC5AC mucins purified from Calu-3 cells grown at ANLI (untreated) and after treatment with an anaerobic bacterial community (ABC, treated). (B) Area under curve (AUC) for both peak 1 (high molecular weight mucins) and peak 2 (low molecular weight mucin). Data shown were derived from three independent experiments using three biological replicates $(n=9)$. Data were compared using a unpaired t-test with Welch's correction $\left.{ }^{* *}, p<.01\right)$.

Anaerobes promote $\boldsymbol{P}$. aeruginosa colonization of the airway epithelium. Recent work has shown that viral challenge of the respiratory epithelium potentiates colonization by $P$. aeruginosa via interferon mediated effects (20). Other work has shown that the protective role of the mucus barrier is compromised by Streptococcus mitis through hydrolysis of mucin glycans (33). Given that the anaerobes used in our model both elicited an inflammatory response and altered mucin integrity, we hypothesized that in addition to providing nutrients for pathogen growth through cross-feeding (17), anaerobic microbiota enhance $P$. aeruginosa colonization of the airway epithelium (depicted in Figure 6A).

To test this hypothesis, Calu-3 cells were first treated with the anaerobic bacterial consortium (ABC) for $24 \mathrm{~h}$, washed to remove spent medium and unbound cells, and subsequently infected with $1 \times 10^{6}$ CFUs of $P$. aeruginosa PA14 for $2 \mathrm{~h}$. Removal of spent medium and a short incubation time ensures that any difference in colonization between anaerobe-treated cells and an unconditioned (i.e., no anaerobe) control was a result of cell attachment and not enhanced growth. $P$. aeruginosa colonization was then determined 
by washing and permeabilization of Calu-3 cells followed by plate enumeration. As predicted, anaerobe pre-treatment resulted in an increase in $1.1 \times 10^{7} \mathrm{P}$. aeruginosa CFUs relative to untreated (i.e., no $A B C$ ) controls $(p=0.0003$, Figure 6B).

To further demonstrate that enhanced pathogen colonization was due to mucin degradation and not some other unidentified process, mucins isolated from Calu-3 cells treated with ABC (and untreated controls) were used to coat the surface of a microtiter plate (34), followed by addition of $P$. aeruginosa PA14. As expected (35), mucin coating led to a 1.5 log-reduction in bacterial attachment relative to uncoated plates (PBS alone). Consistent with our ANLI co-culture assay, ABC-treated mucins resulted in a significant increase $(p=0.0007)$ in $P$. aeruginosa binding compared to untreated mucins (Figure 6C). To confirm that our mucin purification process (e.g., use of guanidine hydrochloride) did not affect $P$. aeruginosa viability, plates were also coated with mucins degraded with human neutrophil elastase (NE) and isolated using the same process. NE-treated mucins resulted in similar PA14 attachment to PBS controls, confirming bacterial viability and that anaerobe-microbiota can enhance pathogen colonization of a mucin-coated interface.

Finally, to assess the contributions of individual anaerobes to $P$. aeruginosa colonization we challenged Calu-3 cells with representative isolates of the three most abundant genera in the anaerobic consortium (Streptococcus, Veillonella, Prevotella) prior to $P$. aeruginosa colonization (Figure 6D). Contrary to a recent study (33), we found that Streptococcus species (S. gordonii and S. parasanguinis) had little effect on PA14 colonization, despite their known mucin degradation capacity. Similarly, V. parvula resulted in no significant differences between treatment conditions. By contrast, challenge with both $P$. melaninogenica and $P$. oris, two species commonly associated with inflammatory airway disease, resulted in significantly increased PA14 recovery from Calu-3 cells compared to unconditioned controls $(p=0.0003$ and $p=0.0004$, respectively). These data demonstrate that while anaerobic microbiota of the respiratory tract likely facilitate enhanced colonization of the airway epithelium via mucin degradation, it is clear they do so in a species-specific manner. 
A

\section{Pre-treatment}

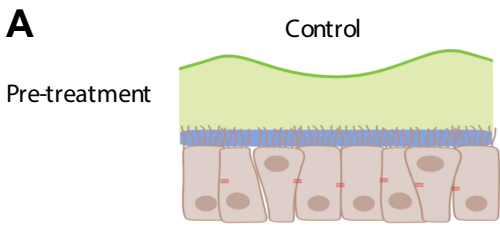

Predicted

P.aeruginosa

treatment

outcome

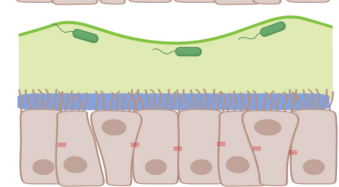

Anaerobes (Individual)
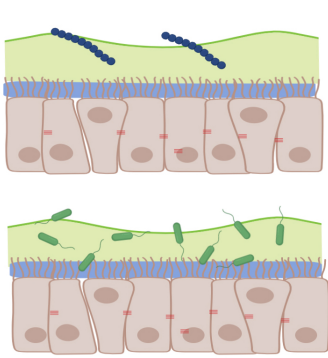

Anaerobes (Consortium)
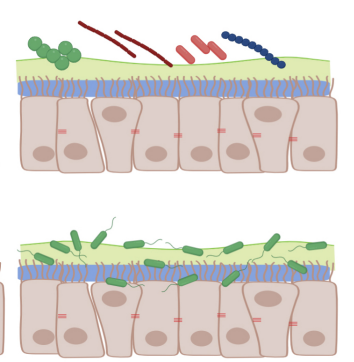
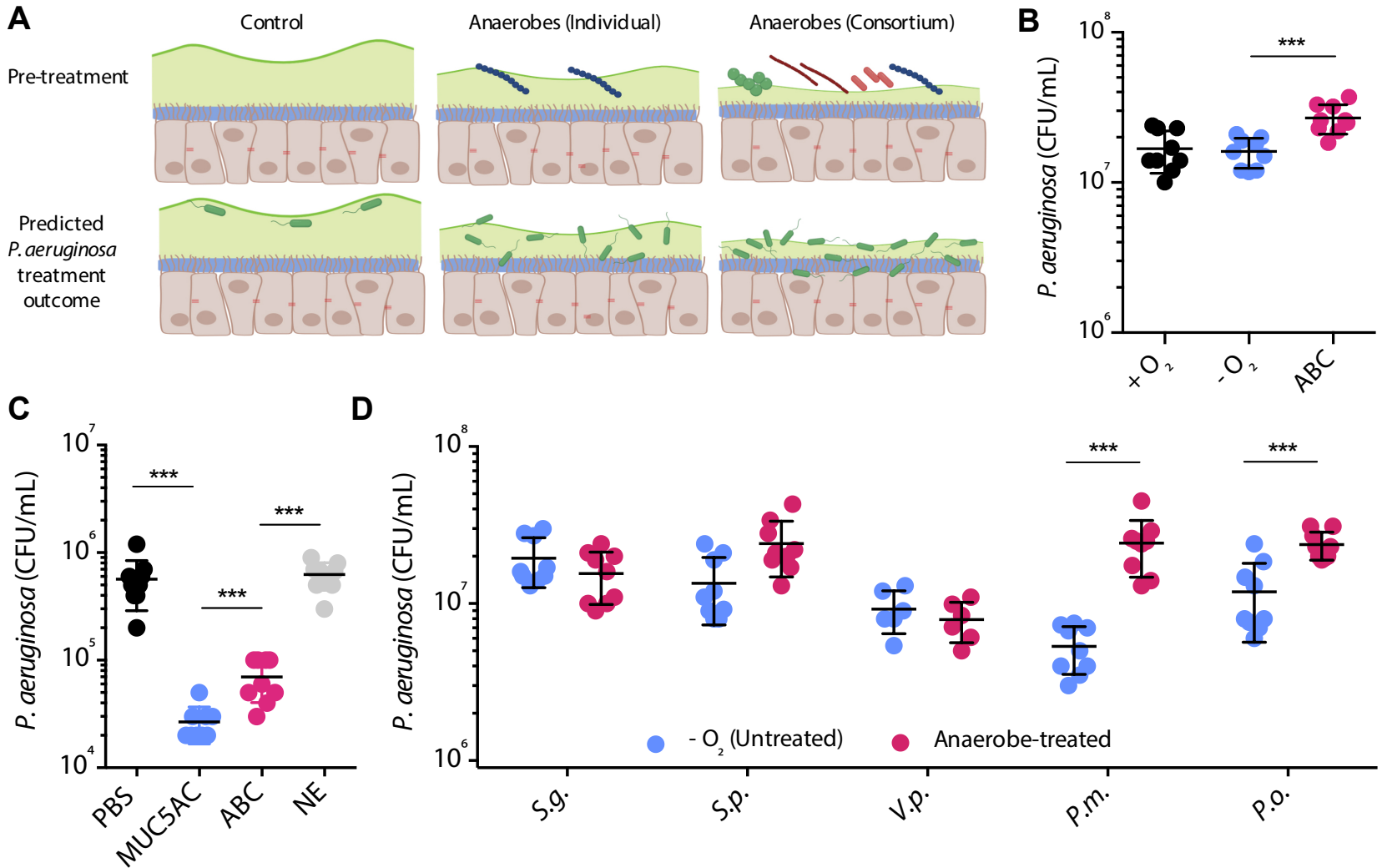

D

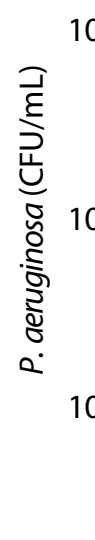

Fig. 6. Anaerobic microbiota condition the epithelial interface for pathogen colonization. (A) Schematic of experimental design. (B) Calu-3 pre-treatment with an anaerobic bacterial consortium (ABC) potentiates $P$. aeruginosa colonization. (C) $P$. aeruginosa adhesion to microtiter plates coated with mucin (MUC5AC), ABC-treated mucin, and neutrophil elastase (NE)-treated mucin relative to an uncoated control (PBS). (D) $P$. aeruginosa adhesion to Calu-3 cells after pretreatment with individual anaerobes (S.g., Streptococcus gordonii; S.p., S. parasanguinis; V.p., Veillonella parvula; P.o., Prevotella oris; P.m., P. melaninogenica). Data shown in panels B-D were derived from three independent experiments with three biological replicates $(n=9)$. Data in $C$ were compared using a one-way ANOVA $(p<0.0001)$ with multiple comparisons. Data in $C$ were compared using a non-parametric Kruskal-Wallace test $(p<0.0001)$ with multiple comparisons. Pairwise comparisons in panel $\mathrm{D}$ were performed using were compared using an unpaired t-test with Welch's correction $\left({ }^{*} p<0.05,{ }^{* * *} p<0001\right)$.

\section{DISCUSSION}

The recent surge in culture-independent sequencing of human microbiota has spawned renewed interest in the importance of anaerobic bacteria in disease etiologies. However, despite anaerobes comprising a significant component of airway bacterial communities, studies on their contributions to disease pathophysiology have reported seemingly contradictory results $(7,19,36-41)$, calling into question their role in patient morbidity. Proposed pathogenic mechanisms are supported by compelling in vitro data $(15-17,42,43)$, but their relevance is unresolved due to a lack of compatible models with which to test 
their interactions with the respiratory epithelium. To address this knowledge gap, here we optimized and characterized a model system that facilitates co-culture of anaerobes and polarized airway epithelial cells. Importantly, provision of oxygen exclusively to the basolateral side of host (Calu-3) cells prevented anoxiaassociated cytotoxicity, inflammation, and significant changes in global gene expression over $24 \mathrm{~h}$, validating its utility for the extended study of anaerobe-host interactions. Using this model, we demonstrate that anaerobic microbiota stimulate an immune response and promote enhanced epithelial colonization by airway pathogens.

Disparate oxygen demands of epithelial cells and anaerobic bacteria pose significant challenges for their co-culture in vitro. Several bacterial-epithelial culture systems have been developed in an attempt to overcome these challenges and recapitulate an oxygen-restricted mucosal interface (44-47), though each has notable drawbacks. Transwell cultures of $\mathrm{Caco}-2, \mathrm{HaCaT}$, and primary human gingival cells have been used to demonstrate that anaerobic taxa can adhere to, invade, and alter oral and intestinal epithelia, yet these assays are either limited to short incubation times or poorly mimic atmospheric conditions observed in vivo (48-51). Newer microfluidic-based and 'organ-on-a-chip' models have also seen widespread interest due to their ability to establish dual oxic-anoxic interfaces and facilitate study of anaerobe-host interactions (52-56). However, these models either preclude direct host-bacterial contact or are technically challenging to maintain both oxic and strict anoxic microcompartments. More recently, new methods have been expanded to include respiratory epithelial cells and used to study alveolar cell infection by M. tuberculosis, but development is still in its infancy (57). Our adapted system offers the distinct advantages of ease-of-use, direct interactions between host and microbiota, reproducibility given the multi-well plate format, and methodological flexibility lending itself to genetic, biochemical, and microscopy studies as demonstrated here. Most notably, this co-culture model elicits few changes in host cell physiology after $24 \mathrm{~h}$ of anoxia, paving the way for a greater understanding of the mechanistic contributions of anaerobic bacteria to airway pathophysiology.

To demonstrate the utility of our model we used a polymicrobial consortium representative of anaerobic bacterial community signatures observed in acute and chronic airway disease $(8,11,39,58)$. Dominated by Streptococcus, Prevotella, and Veillonella spp., this consortium (among other bacterial taxa) 
is thought to seed the airways through microaspiration from the oral cavity and is recognized as a key risk factor in the development of COPD, CF, pneumonia, sinusitis and other diseases. For example, in vitro studies have shown that anaerobe-derived supernatants containing proteases and pro-inflammatory shortchain fatty acids modulate the immune tone of bronchial epithelial cell lines and primary cell cultures $(15$, 16). Indeed, anaerobe abundance in both diseased airways and healthy controls is associated with enhanced expression of inflammatory cytokines $(1,12)$. Our data also support a immunomodulatory role and suggest that colonization of the airway mucosa by aspirated anaerobes may establish a local inflammatory environment known to promote colonization by $P$. aeruginosa and other canonical pathogens $(20,21)$.

We previously reported that anaerobes can also stimulate pathogen growth through mucin-based cross-feeding (17). Specifically, P. aeruginosa, which cannot efficiently catabolize mucins in isolation, can gain access to bioavailable substrates via anaerobe-mediated degradation of the mucin polypeptide and O-linked glycans. Though not directly tested here, it is plausible that pre-colonization with an anaerobic bacterial community liberates additional mucin-derived metabolites on which pathogens can thrive at the epithelial interface. While it remains unclear why only low molecular weight mucins were altered, our FPLC data confirm that the mucosal surface is structurally modified as a result of anaerobic colonization. Given that mucin degradation has been shown to compromise its barrier function and enhance pathogenepithelial interactions (33), we propose that in addition to the host's impaired mucociliary clearance, pathogenic contributions of anaerobic microbiota to airway infection are likely imparted through a multifactorial process (inflammation, cross-feeding, and surface alteration).

By focusing on an early time point after $P$. aeruginosa challenge (2h), we targeted anaerobic mucin degradation and its role in re-shaping the epithelial interface while enhancing pathogen attachment, as was previously shown for S. mitis and Neisseria meningitidis (33). As predicted, anaerobe degradation of apical mucus resulted in a significant increase in $P$. aeruginosa attachment, which has important clinical implications. Most notably, epithelial colonization is known to stimulate rapid $P$. aeruginosa biofilm maturation and associated increases in extracellular polysaccharide production, induction of quorumsensing and other transcriptional changes (22). In addition, $P$. aeruginosa grown on bronchial epithelial 
cells is far more resistant to antibiotic treatment than when grown on abiotic surfaces (22), consistent with their increased tolerance in vivo. We propose that anaerobe-mediated colonization further potentiates these phenotypes. Moving forward, it will be important to consider $P$. aeruginosa interactions with the host over longer time periods to further understand differences in pathogen physiology and the inflammatory host response in the presence and absence of anaerobic microbiota.

We elected to use Calu-3 cells as a representative cell line for several reasons. First, Calu-3 cells reach polarization at ALI within $\sim 21$ days, express high levels of occludin and E-cadherin localized at tight junctions, and achieve TEER values $\left(>1000 \Omega \mathrm{cm}^{2}\right)$ far greater and more stable than those of primary cells (59-61). In addition, Calu-3s express high levels of CFTR (62), demonstrating their suitability and relevance for studies of CF and COPD, for which CFTR-silencing small hairpin RNAs (shRNA) have already been developed (63). Finally, unlike many other respiratory cell lineages, overproduction of mucus on the apical surface of polarized Calu-3 cells mimics a diseased mucosal environment and allowed us to test the hypothesis that mucin degradation enhances pathogen colonization. This was an important consideration as $P$. aeruginosa biofilms, at least in the context of CF, are thought to form within secreted mucus as opposed to the epithelial layer $(64,65)$. We also acknowledge limitations. Unlike primary cells, which form a pseudostratified epithelium with mucociliary differentiation, Calu-3 cells are derived from human bronchial submucosal glands that comprise a relatively homogenous monolayer. Transcriptional and physiological responses to external stimuli may also be unique to Calu-3s. As an example, $S$. aureus enterotoxin B is known to elicit significant differences in barrier integrity, IL-6 and IL-8 production relative to primary tissue (66). These, among other distinctions, underscore the importance of future work testing additional cell lines and primary tissue.

Despite these limitations, our model represents a tractable co-culture system that facilitates extended interrogation of host-anaerobe interactions. While we use this model here to demonstrate a role for anaerobic microbiota in the initial colonization of the epithelial surface, this work will undoubtedly benefit future studies focused on anaerobe-host and anaerobe-host-pathogen interactions and their dynamics over time. Not only do we anticipate generating a deeper understanding of $P$. aeruginosa pathophysiology under oxygen-limited conditions, we also expect to identify new therapeutic strategies in addition to 
understanding how existing antimicrobials are impacted by environmental conditions known to exist in vivo (64). Finally, while we use an airway-derived bacterial community and $P$. aeruginosa as our model organisms, this work motivates additional studies of the gut, lung, oral cavity, and other sites of infection, where etiological roles of anaerobes have been proposed but specific pathogenic mechanisms remain unclear.

\section{METHODS}

Epithelial Cell Culture. Calu-3 cells were maintained in Minimal Essential Medium (Corning, USA) in 10\% fetal bovine serum (FBS, Gene) supplemented with $100 \mathrm{U} / \mathrm{mL}$ penicillin and $100 \mu \mathrm{g} / \mathrm{mL}$ streptomycin (Gibco) at $37^{\circ} \mathrm{C}$ in a $5 \% \mathrm{CO}_{2}$ incubator. Upon reaching $80 \%$ confluency, $1 \times 10^{5}$ cells were passaged onto $6.5 \mathrm{~mm}$ culture inserts (12-well hanging inserts, $0.4 \mu \mathrm{m}$ pore; Corning). When cells reached confluency ( $\sim 5$ days), apical medium was removed to establish an air-liquid interface (ALI). Polarized cells were maintained for an additional 21-28 days to facilitate differentiation and mucus accumulation.

Cell cultures were then assembled in a gas permeable multi-well plate manifold based on an enteroid-anaerobe co-culture system recently described (23) (Figure S2). Briefly, once polarized, Calu-3 cells were placed into gaskets in a 24-well gas-permeable plate (CoyLabs, Grass Lake, MI) containing $800 \mu \mathrm{l}$ of MEM per well. Mineral oil $(400 \mu \mathrm{L})$ was added to unused wells to prevent gas permeation from the basolateral to apical side of the manifold. Once assembled, the apparatus was moved to an anaerobic chamber $\left(90 \% \mathrm{~N}_{2} / 5 \% \mathrm{H} 2 / 5 \% \mathrm{CO}_{2}\right)$ while mixed gas $\left(21 \% \mathrm{O}_{2} / 5 \% \mathrm{CO}_{2} / 74 \% \mathrm{~N}_{2}\right)$ was delivered to the base of the plate to oxygenate the basolateral side of the polarized monolayer. A schematic of this workflow is summarized in Figure 1.

Cell culture assays. Calu-3 barrier integrity was determined by trans-epithelial electrical resistance (TEER) measured with a Millicell-ERS2 Volt-Ohm meter (Millipore Sigma). Barrier integrity was further assessed using the Human E-cadherin Quantikine enzyme-linked immunosorbent assay (ELISA) kit (R\&D Systems, Minneapolis, MN). Absorbance was measured at $450 \mathrm{~nm}$ using a BioTek Synergy H2 plate reader and concentrations of E-cadherin were determined against a standard curve according to 
manufacturer's instructions. Tight junction formation was assayed using immunofluorescence with an antizona occludens (ZO)-1 monoclonal antibody (Thermo). To do so, Calu-3 cultures on Transwell inserts were chemically fixed in $4 \%$ paraformaldehyde (PFA) in phosphate-buffered saline (PBS) for $1 \mathrm{~h}$ at room temperature. Cells were blocked in 10\% goat serum and 1\% bovine serum albumin (Sigma) for 15 min. After blocking, cells were incubated with human anti-mouse ZO-1 (AlexaFluor-488 conjugate; ThermoFisher)( $(5 \mu \mathrm{g} / \mathrm{mL})$ for $1 \mathrm{~h}$. Transwell membranes were washed, removed from the supporting plastic insert, and mounted on glass slides using Vectashield anti-fade mounting medium. Labeled cells were visualized (ex. 480nm, em. 525nm) on an Olympus IX83 inverted fluorescence microscope using a 20X objective lens $(0.75 \mathrm{NA})$. Finally, cytotoxicity was determined by lactate dehydrogenase measurements on cell-free supernatants using the Cytotoxicity Detection Kit Plus assay (Roche) according to manufacturer's instructions. Data were calculated as percent LDH release compared with a lysed control and reported as $\% \mathrm{LDH}$ release $=[($ experimental value low control $) /($ high control-low control $)] \times 100$.

Immunoassays. The pro-inflammatory cytokine response of Calu-3 cells was measured after $24 \mathrm{~h}$ of culture in the anaerobic chamber. To do so, spent medium was collected from the apical side of Transwell cultures, and tumor necrosis factor alpha (TNF- $\alpha$ ), interleukin (IL-) 8 and IL-6 were then measured by ELISA per manufacturer instructions (R\&D systems). Cells grown under standard incubator conditions (5\% $\mathrm{CO}_{2}$ ) were used as a control.

FPLC. Secreted mucins were collected from Calu-3 cells as previously described (24). Briefly, cells grown on Transwell inserts were solubilized in a reduction buffer consisting of $6 \mathrm{M}$ guanidine hydrochloride, $0.1 \mathrm{M}$ Tris-HCl buffer, and 5mM EDTA (pH 8). Prior to solubilization, 10mM dithiothreitol (DTT) and a cOmplete Mini protease inhibitor tablet (Roche) were added to $400 \mathrm{~mL}$ of reduction buffer to minimize mucin degradation. Cell suspensions were gently agitated by pipetting to dislodge biomass, and each of the six Transwell suspensions per plate were pooled into a single aliquot. Cells were rinsed with a reduction buffer to remove residual mucin. This mixture was then incubated for $5 \mathrm{~h}$ at $37^{\circ} \mathrm{C}$, followed by the addition of 25 $\mathrm{mM}$ iodoacetamide and incubation overnight at room temperature. Mucins were then dialyzed (1000 kDa 
MWCO) against $1 \mathrm{~L}$ of $4 \mathrm{M} \mathrm{GuHCl}$ buffer containing $2.25 \mathrm{mM} \mathrm{NaH} \mathrm{PO}_{4}-\mathrm{H}_{2} \mathrm{O}$ and $76.8 \mathrm{mM} \mathrm{Na}_{2} \mathrm{HPO}_{4}$ and proceeded for $36 \mathrm{~h}$ with buffer exchanges every $12 \mathrm{~h}$.

Fast protein liquid chromatography (FPLC) size-exclusion chromatography was then used to evaluate the integrity of high-molecular weight mucins. Using an Akta Pure FPLC (GE Healthcare BioSciences, Marlborough, MA) housed at $4^{\circ} \mathrm{C}, 500 \mu \mathrm{L}$ of purified mucin was manually injected and subjected to an isocratic run at a flow rate of $0.4 \mathrm{~mL} / \mathrm{min}$ for 1.5 column volumes (CV) with $150 \mathrm{mM} \mathrm{NaCl}$ in $50 \mathrm{mM}$ phosphate buffer $(\mathrm{pH} 7.2)$ on a 15mL 10/200 Tricorn column packed with Sepharose 4B-CL beads. Data were collected using Unicorn 7 software (GE Healthcare Biosciences).

Bacterial strains and culture conditions. P. aeruginosa PA14 was obtained from D.K. Newman (California Institute of Technology) and was routinely cultured on Luria Bertani (LB) medium. $P$. melaninogenica ATCC 25845, S. parasanguinis ATCC15912, and V. parvula ATCC10790 were obtained from Microbiologics (St. Cloud, MN). S. gordonii was obtained from M.C. Herzberg (University of Minnesota) and P. oris 12252T was purchased from the Japan Collection of Microorganisms. All anaerobes were maintained on Brain-Heart Infusion medium supplemented with hemin $(0.25 \mathrm{~g} / \mathrm{L})$, vitamin $\mathrm{K}(0.025$ $\mathrm{g} / \mathrm{L})$ and laked sheep's blood (5\% vol/vol) $(\mathrm{BHI}-\mathrm{HKB})$ in an anaerobic chamber. A mucin-enriched anaerobic bacterial community $(\mathrm{ABC})$ derived from an individual with chronic rhinosinusitis was also used and was cultured in a minimal mucin medium (MMM) described previously (17).

Bacterial challenge and infection. Forty-eight hours prior to bacterial challenge, Calu-3 cells were incubated in MEM/FBS without antibiotics. On the day of bacterial challenge, Transwells were assembled in the gas permeable culture system and transferred into the anaerobic chamber where they were allowed to equilibrate for $3 \mathrm{~h}$. Overnight cultures of each anaerobe and the anaerobic community $(\mathrm{ABC})$ were grown in MMM. Each individual culture was diluted to a concentration of $\sim 1 \times 10^{6}$ colony forming units (CFU) in MEM and $10 \mu \mathrm{L}$ of bacterial suspension was added to the apical side of the Calu-3 cells. Similarly, $10 \mu \mathrm{L}$ of an adjusted suspension (OD600nm $=0.1$ ) of the anaerobic community was added to separate wells. Co-cultures were then incubated for an additional $24 \mathrm{~h}$. Following anaerobe challenge, spent medium was collected and analyzed for cytotoxicity using the LDH colorimetric assay (described above). Mucins were 
also collected as described above for integrity analysis via FPLC. In a separate experiment, anaerobe viability was determined using plate enumeration. Briefly, Calu-3 cells were washed with $100 \mu \mathrm{L}$ of PBS (to remove loosely bound cells) or $0.25 \%$ Triton X-100 (to recover tightly bound or intracellular bacteria). Resulting washes were serially diluted and plated on $\mathrm{BHI}$ or Brucella Laked Blood Agar (BBA) with Kanamycin $(100 \mu \mathrm{g} / \mathrm{mL})$ and Vancomycin $(7.5 \mu \mathrm{g} / \mathrm{mL})$ for enumeration.

For the $P$. aeruginosa colonization assay, Calu-3 cultures were removed from the anaerobic chamber following anaerobe challenge. Cells were gently washed with PBS and subsequently infected with $1 \times 10^{6} \mathrm{CFU}$ of $P$. aeruginosa for $2 \mathrm{~h}$. After incubation, cells were gently washed three times with PBS to remove unbound $P$. aeruginosa and were permeabilized using $0.25 \%$ Triton $\mathrm{X}-100$. Bacteria were enumerated by plating serial dilutions of Calu-3 cell lysates on LB agar. All assays were performed using three biological replicates and data are reported as the mean of three experiments.

RNA sequencing. The transcriptomic response of Calu- 3 cells to anoxic culture and anaerobe challenge was determined using RNAseq. Calu-3 cells were cultured at ALI as described above and harvested after $24 \mathrm{~h}$ of anaerobic incubation in the presence/absence of bacterial challenge. Normoxic (unchallenged) cells were maintained under standard incubator conditions. At the conclusion of each experiment, RNAlater (Invitrogen) was added to the apical and basolateral side of each well. For each condition, RNA was isolated from 5 or 6 Transwells using the RNeasy Micro Plus kit (Qiagen) according to manufacturer's instructions. DNase treatment was performed as part of the RNA Clean and Concentrator kit (Zymo). RNA quality (RIN > 9.7) and quantity were assessed using an Agilent Bioanalyzer and RiboGreen, respectively. cDNA libraries were prepared using the SMARTer Universal Low Input RNA Kit (Takara Bio) and submitted for sequencing at the University of Minnesota Genomics Center on the Illumina NovaSeq 6000 platform.

The Ensembl GTF annotation file was filtered to remove annotations for non-protein-coding features. Fastq files were evenly subsampled down to a maximum of 100,000 reads per sample. Data quality in fastq files was assessed with FastQC. Raw reads were mapped to reference Human (Homo_sapiens) genome assembly "GRCh38" using annotation from Ensembl release 98. Gene counts were generated with 'featureCounts' of the RSubread package (67). DESeq2/1.28.1 was used to estimate size factors to 
464

465

466

467

468

469

470

471

472

473

474

475

476

477

478

479

480

481

482

483

484

485

486

487

generate normalized count data, estimate gene-wise dispersions, shrink estimates using type='ashr', and perform Wald hypothesis testing $(68,69)$. Genes with a $\log _{2}$ fold-change greater than 1 and BenjaminiHochberg adjusted $p$-value $<0.001$ were considered significant. Code and data files are shared at https://github.com/Hunter-Lab-UMN/Moore PJ 2020.

Scanning electron microscopy. Untreated, anaerobe-challenged, and P. aeruginosa-infected cell cultures were washed three times in $0.2 \mathrm{M}$ sodium cacodylate buffer, and submerged in primary fixative ( $0.15 \mathrm{M}$ sodium cacodylate buffer, $\mathrm{pH} 7.4,2 \%$ paraformaldehyde, $2 \%$ glutaraldehyde, $4 \%$ sucrose, $0.15 \%$ alcian blue $8 \mathrm{GX}$ ) for $22 \mathrm{~h}$. Transwell membranes were washed three more times prior to a 90 minute treatment with secondary fixative ( $1 \%$ osmium tetroxide, $1.5 \%$ potassium ferrocyanide, $0.135 \mathrm{M}$ sodium cacodylate, $\mathrm{pH} 7.4)$. After three final washes, cells were dehydrated in a graded ethanol series $(25 \%, 50 \%$, $75 \%, 85 \%, 2 \times 95 \%$, and $2 \times 100 \%$ ) for 10 minutes each before $\mathrm{CO}_{2}$-based critical point drying. Transwell membranes were attached to SEM specimen mounts using carbon conductive adhesive tape and sputter coated with $\sim 5 \mathrm{~nm}$ iridium using the Leica ACE 600 magnetron-based system. Cells were imaged using a Hitachi S-4700 field emission SEM with an operating voltage of 2kV. Images were false colored using Adobe Photoshop CS6.

Microtiter plate binding assay. $P$. aeruginosa adhesion to mucus was tested using an established microtiter plate-based assay (34). 96 well MaxiSorp microtiter plates (Nunc) were coated with $40 \mu \mathrm{g} / \mathrm{ml}$ of mucins (MUC5AC) derived from untreated and ABC-treated Calu-3 cells. As a control, MUC5AC treated with neutrophil elastase $(1 \mu \mathrm{g} / \mathrm{mL})$ was also used. After coating, plates were incubated for $24 \mathrm{~h}$ at $37^{\circ} \mathrm{C}$. Mucin-coated wells were then washed three times with sterile PBS to remove any residual unbound mucin. $1 \times 10^{6} \mathrm{CFUs}$ of $P$. aeruginosa PA14 were added to mucin-coated wells and incubated for an additional $2 \mathrm{~h}$ at $37^{\circ} \mathrm{C}$. Wells were washed 10 times with PBS to remove any unbound bacteria. Bound PA14 was desorbed by treatment with $200 \mu$ l of $0.25 \%$ Triton X-100 for $15 \mathrm{~min}$ at room temperature. Bacteria bound to each well were enumerated by plating serial dilutions on LB agar. All assays were performed using three biological replicates and data are reported as the mean of three experiments $(n=9)$. 


\section{REFERENCES}

490

491

492

493

494

495

496

497

498

499

500

501

502

503

504

505

506

507

508

509

510

511

512

513

514

515

516

517

518

519

520

521

522

523

1. L. N. Segal, A. V. Alekseyenko, J. C. Clemente, R. Kulkarni, B. Wu, Z. Gao, H. Chen, K. I. Berger, R. M. Goldring, W. N. Rom, M. J. Blaser, M. D. Weiden, Enrichment of lung microbiome with supraglottic taxa is associated with increased pulmonary inflammation. Microbiome 1, 19 (2013).

2. E. S. Charlson, K. Bittinger, A. R. Haas, A. S. Fitzgerald, I. Frank, A. Yadav, F. D. Bushman, R. G. Collman, Topographical continuity of bacterial populations in the healthy human respiratory tract. $A m$ J Respir Crit Care Med 184, 957-963 (2011).

3. A. Morris, J. M. Beck, P. D. Schloss, T. B. Campbell, K. Crothers, J. L. Curtis, S. C. Flores, A. P. Fontenot, E. Ghedin, L. Huang, K. Jablonski, E. Kleerup, S. V. Lynch, E. Sodergren, H. Twigg, V. B. Young, C. M. Bassis, A. Venkataraman, T. M. Schmidt, G. M. Weinstock, L. H. M. Project, Comparison of the respiratory microbiome in healthy nonsmokers and smokers. Am J Respir Crit Care Med 187, 1067-1075 (2013).

4. R. P. Dickson, J. R. Erb-Downward, C. M. Freeman, L. McCloskey, N. R. Falkowski, G. B. Huffnagle, J. L. Curtis, Bacterial topography of the healthy human lower respiratory tract. mBio 8, (2017).

5. A. A. Pragman, H. B. Kim, C. S. Reilly, C. Wendt, R. E. Isaacson, The lung microbiome in moderate and severe chronic obstructive pulmonary disease. PLoS One 7, e47305 (2012).

6. Y. J. Huang, E. Kim, M. J. Cox, E. L. Brodie, R. Brown, J. P. Wiener-Kronish, S. V. Lynch, A persistent and diverse airway microbiota present during chronic obstructive pulmonary disease exacerbations. OMICS 14, 9-59 (2010).

7. M. M. Tunney, T. R. Field, T. F. Moriarty, S. Patrick, G. Doering, M. S. Muhlebach, M. C. Wolfgang, R. Boucher, D. F. Gilpin, A. McDowell, J. S. Elborn, Detection of anaerobic bacteria in high numbers in sputum from patients with cystic fibrosis. Am J Respir Crit Care Med 177, 995-1001 (2008).

8. G. B. Rogers, C. J. van der Gast, L. Cuthbertson, S. K. Thomson, K. D. Bruce, M. L. Martin, D. J. Serisier, Clinical measures of disease in adult non-CF bronchiectasis correlate with airway microbiota composition. Thorax 68, 731-737 (2013).

9. J. G. Bartlett, The role of anaerobic bacteria in lung abscess. Clin Infect Dis 40, 923-925 (2005).

10. S. K. Lucas, R. Yang, J. M. Dunitz, H. C. Boyer, R. C. Hunter, $16 S$ rRNA gene sequencing reveals site-specific signatures of the upper and lower airways of cystic fibrosis patients. $J$ Cyst Fibros 17, 204-212 (2018).

11. P. L. Molyneaux, S. A. G. Willis-Owen, M. J. Cox, P. James, S. Cowman, M. Loebinger, A. Blanchard, L. M. Edwards, C. Stock, C. Daccord, E. A. Renzoni, A. U. Wells, M. F. Moffatt, W. O. C. Cookson, T. M. Maher, Host-microbial interactions in idiopathic pulmonary fibrosis. Am J Respir Crit Care Med 195, 1640-1650 (2017).

12. L. N. Segal, J. C. Clemente, Y. Li, C. Ruan, J. Cao, M. Danckers, A. Morris, S. Tapyrik, B. G. Wu, P. Diaz, G. Calligaro, R. Dawson, R. N. van Zyl-Smit, K. Dheda, W. N. Rom, M. D. Weiden, Anaerobic 
bacterial fermentation products increase tuberculosis risk in antiretroviral-drug-treated HIV patients. Cell Host Microbe 21, 530-537.e534 (2017).

13. E. T. Zemanick, B. D. Wagner, S. D. Sagel, M. J. Stevens, F. J. Accurso, J. K. Harris, Reliability of quantitative real-time PCR for bacterial detection in cystic fibrosis airway specimens. PLoS One 5, e15101 (2010).

14. I. Sulaiman, B. G. Wu, Y. Li, A. S. Scott, P. Malecha, B. Scaglione, J. Wang, A. Basavaraj, S. Chung, K. Bantis, J. Carpenito, J. C. Clemente, N. Shen, J. Bessich, S. Rafeq, G. Michaud, J. Donington, C. Naidoo, G. Theron, G. Schattner, S. Garofano, R. Condos, D. Kamelhar, D. Addrizzo-Harris, L. N. Segal, Evaluation of the airway microbiome in nontuberculous mycobacteria disease. Eur Respir J 52, (2018).

15. B. Mirković, M. A. Murray, G. M. Lavelle, K. Molloy, A. A. Azim, C. Gunaratnam, F. Healy, D. Slattery, P. McNally, J. Hatch, M. Wolfgang, M. M. Tunney, M. S. Muhlebach, R. Devery, C. M. Greene, N. G. McElvaney, The role of short-chain fatty acids, produced by anaerobic bacteria, in the cystic fibrosis airway. Am J Respir Crit Care Med 192, 1314-1324 (2015).

16. P. Ghorbani, P. Santhakumar, Q. Hu, P. Djiadeu, T. M. Wolever, N. Palaniyar, H. Grasemann, Shortchain fatty acids affect cystic fibrosis airway inflammation and bacterial growth. Eur Respir J 46, 10331045 (2015).

17. J. M. Flynn, D. Niccum, J. M. Dunitz, R. C. Hunter, Evidence and role for bacterial mucin degradation in cystic fibrosis airway disease. PLoS Pathog 12, e1005846 (2016).

18. J. M. Flynn, L. C. Cameron, T. D. Wiggen, J. M. Dunitz, W. R. Harcombe, R. C. Hunter, Disruption of cross-feeding inhibits pathogen growth in the sputa of patients with cystic fibrosis. mSphere 5, (2020).

19. L. A. Carmody, L. J. Caverly, B. K. Foster, M. A. M. Rogers, L. M. Kalikin, R. H. Simon, D. R. VanDevanter, J. J. LiPuma, Fluctuations in airway bacterial communities associated with clinical states and disease stages in cystic fibrosis. PLoS One 13, e0194060 (2018).

20. M. R. Hendricks, L. P. Lashua, D. K. Fischer, B. A. Flitter, K. M. Eichinger, J. E. Durbin, S. N. Sarkar, C. B. Coyne, K. M. Empey, J. M. Bomberger, Respiratory syncytial virus infection enhances Pseudomonas aeruginosa biofilm growth through dysregulation of nutritional immunity. Proc Natl Acad Sci U S A 113, 1642-1647 (2016).

21. M. R. Kiedrowski, J. R. Gaston, B. R. Kocak, S. L. Coburn, S. Lee, J. M. Pilewski, M. M. Myerburg, J. M. Bomberger, Biofilm growth on cystic fibrosis airway epithelial cells Is enhanced during respiratory syncytial virus coinfection. mSphere 3, (2018).

22. S. Moreau-Marquis, J. M. Bomberger, G. G. Anderson, A. Swiatecka-Urban, S. Ye, G. A. O'Toole, B. A. Stanton, The DeltaF508-CFTR mutation results in increased biofilm formation by Pseudomonas aeruginosa by increasing iron availability. Am J Physiol Lung Cell Mol Physiol 295, L25-37 (2008). 
23 T. Fofanova, C. Stewart, J. Auchtung, R. Wilson, R. Britton, K. Grande-Allen, M. Estes, J. Petrosino, A novel human enteroid-anaerobe co-culture system to study microbial-host interaction under physiological hypoxia. bioRxiv 555755 (2019).

24. C. Evert, T. Loesekann, G. Bhat, A. Shajahan, R. Sonon, P. Azadi, R. C. Hunter, Generation of 13Clabeled MUC5AC mucin oligosaccharides for stable isotope probing of host-associated microbial communities. ACS Infect Dis 5, 385-393 (2019).

25. M. Tamm, M. Bihl, O. Eickelberg, P. Stulz, A. P. Perruchoud, M. Roth, Hypoxia-induced interleukin-6 and interleukin-8 production is mediated by platelet-activating factor and platelet-derived growth factor in primary human lung cells. Am J Respir Cell Mol Biol 19, 653-661 (1998).

26. L. Jia, Y. Shi, Y. Wen, W. Li, J. Feng, C. Chen, The roles of TNFAIP2 in cancers and infectious diseases. J Cell Mol Med 22, 5188-5195 (2018).

27. K. De Filippo, A. Dudeck, M. Hasenberg, E. Nye, N. van Rooijen, K. Hartmann, M. Gunzer, A. Roers, N. Hogg, Mast cell and macrophage chemokines CXCL1/CXCL2 control the early stage of neutrophil recruitment during tissue inflammation. Blood 121, 4930-4937 (2013).

28. E. K. Koltsova, K. Ley, The mysterious ways of the chemokine CXCL5. Immunity 33, 7-9 (2010).

29. E. M. Peterman, C. Sullivan, M. F. Goody, I. Rodriguez-Nunez, J. A. Yoder, C. H. Kim, Neutralization of mitochondrial superoxide by superoxide dismutase 2 promotes bacterial clearance and regulates phagocyte numbers in zebrafish. Infect Immun 83, 430-440 (2015).

30. J. M. Sallenave, G. A. Cunningham, R. M. James, G. McLachlan, C. Haslett, Regulation of pulmonary and systemic bacterial lipopolysaccharide responses in transgenic mice expressing human elafin. Infect Immun 71, 3766-3774 (2003).

31. A. G. Henderson, C. Ehre, B. Button, L. H. Abdullah, L. H. Cai, M. W. Leigh, G. C. DeMaria, H. Matsui, S. H. Donaldson, C. W. Davis, J. K. Sheehan, R. C. Boucher, M. Kesimer, Cystic fibrosis airway secretions exhibit mucin hyperconcentration and increased osmotic pressure. J Clin Invest 124, 30473060 (2014).

32. D. J. Thornton, J. R. Davies, S. Kirkham, A. Gautrey, N. Khan, P. S. Richardson, J. K. Sheehan, Identification of a nonmucin glycoprotein (gp-340) from a purified respiratory mucin preparation: evidence for an association involving the MUC5B mucin. Glycobiology 11, 969-977 (2001).

33. M. Audry, C. Robbe-Masselot, J. P. Barnier, B. Gachet, B. Saubaméa, A. Schmitt, S. SchönherrHellec, R. Léonard, X. Nassif, M. Coureuil, Airway mucus restricts neisseria meningitidis away from nasopharyngeal epithelial cells and protects the mucosa from inflammation. $m$ Sphere 4, (2019).

34. S. Vishwanath, R. Ramphal, Adherence of Pseudomonas aeruginosa to human tracheobronchial mucin. Infect Immun 45, 197-202 (1984).

35. J. Y. Co, G. Cárcamo-Oyarce, N. Billings, K. M. Wheeler, S. C. Grindy, N. Holten-Andersen, K. Ribbeck, Mucins trigger dispersal of. NPJ Biofilms Microbiomes 4, 23 (2018). 
593

594

595

596

597

598

599

600

601

602

603

604

605

606

607

608

609

610

611

612

613

614

615

616

617

618

619

620

621

622

623

624

625

626

627

628

36. M. Ulrich, I. Beer, P. Braitmaier, M. Dierkes, F. Kummer, B. Krismer, U. Schumacher, U. GräplerMainka, J. Riethmüller, P. Ø. Jensen, T. Bjarnsholt, N. Høiby, G. Bellon, G. Döring, Relative contribution of Prevotella intermedia and Pseudomonas aeruginosa to lung pathology in airways of patients with cystic fibrosis. Thorax 65, 978-984 (2010).

37. E. T. Zemanick, B. D. Wagner, C. E. Robertson, R. C. Ahrens, J. F. Chmiel, J. P. Clancy, R. L. Gibson, W. T. Harris, G. Kurland, T. A. Laguna, S. A. McColley, K. McCoy, G. Retsch-Bogart, K. T. Sobush, P. L. Zeitlin, M. J. Stevens, F. J. Accurso, S. D. Sagel, J. K. Harris, Airway microbiota across age and disease spectrum in cystic fibrosis. Eur Respir J 50, (2017).

38. R. A. Quinn, K. Whiteson, Y. W. Lim, P. Salamon, B. Bailey, S. Mienardi, S. E. Sanchez, D. Blake, D. Conrad, F. Rohwer, A Winogradsky-based culture system shows an association between microbial fermentation and cystic fibrosis exacerbation. ISME J 9, 1052 (2015).

39. R. Raghuvanshi, K. Vasco, Y. Vázquez-Baeza, L. Jiang, J. T. Morton, D. Li, A. Gonzalez, L. DeRight Goldasich, G. Humphrey, G. Ackermann, A. D. Swafford, D. Conrad, R. Knight, P. C. Dorrestein, R. A. Quinn, High-resolution longitudinal dynamics of the cystic fibrosis sputum microbiome and metabolome through antibiotic therapy. mSystems 5, (2020).

40. E. Jubinville, M. Veillette, J. Milot, F. Maltais, A. M. Comeau, R. C. Levesque, C. Duchaine, Exacerbation induces a microbiota shift in sputa of COPD patients. PLoS One 13, e0194355 (2018).

41. K. Yamasaki, H. Mukae, T. Kawanami, K. Fukuda, S. Noguchi, K. Akata, K. Naito, K. Oda, T. Ogoshi, C. Nishida, T. Orihashi, Y. Kawanami, H. Ishimoto, H. Taniguchi, K. Yatera, Possible role of anaerobes in the pathogenesis of nontuberculous mycobacterial infection. Respirology 20, 758-765 (2015).

42. A. Venkataraman, M. A. Rosenbaum, J. J. Werner, S. C. Winans, L. T. Angenent, Metabolite transfer with the fermentation product 2,3-butanediol enhances virulence by Pseudomonas aeruginosa. ISME J 8, 1210-1220 (2014).

43. J. Phan, T. Gallagher, A. Oliver, W. E. England, K. Whiteson, Fermentation products in the cystic fibrosis airways induce aggregation and dormancy-associated expression profiles in a CF clinical isolate of Pseudomonas aeruginosa. FEMS Microbiol Lett 365, (2018).

44. J. Z. H. von Martels, M. Sadaghian Sadabad, A. R. Bourgonje, T. Blokzijl, G. Dijkstra, K. N. Faber, H. J. M. Harmsen, The role of gut microbiota in health and disease: In vitro modeling of host-microbe interactions at the aerobe-anaerobe interphase of the human gut. Anaerobe 44, 3-12 (2017).

45. G.-S. Park, M. H. Park, W. Shin, C. Zhao, S. Sheikh, S. J. Oh, H. J. Kim, Emulating host-microbiome ecosystem of human gastrointestinal tract in vitro. Stem Cell Reviews and Reports 13, 321-334 (2017).

46. M. Yee, S. Kim, P. Sethi, N. Düzgüneş, K. Konopka, Porphyromonas gingivalis stimulates IL-6 and IL8 secretion in GMSM-K, HSC-3 and H413 oral epithelial cells. Anaerobe 28, 62-67 (2014).

47. A. Pinnock, C. Murdoch, K. Moharamzadeh, S. Whawell, C. W. I. Douglas, Characterisation and optimisation of organotypic oral mucosal models to study Porphyromonas gingivalis invasion. Microbes and Infection 16, 310-319 (2014). 
629

630

631

632

633

634

635

636

637

638

639

640

641

642

643

644

645

646

647

648

649

650

651

652

653

654

655

656

657

658

659

660

661

662

663

48. Y. W. Han, W. Shi, G. T. Huang, S. Kinder Haake, N. H. Park, H. Kuramitsu, R. J. Genco, Interactions between periodontal bacteria and human oral epithelial cells: Fusobacterium nucleatum adheres to and invades epithelial cells. Infection and immunity 68, 3140-3146 (2000).

49. U. K. Gursoy, E. Könönen, V. J. Uitto, Prevotella intermedia ATCC 25611 targets host cell lamellipodia in epithelial cell adhesion and invasion. Oral Microbiology and Immunology 24, 304-309 (2009).

50. D. Ulluwishewa, R. C. Anderson, W. Young, W. C. McNabb, P. van Baarlen, P. J. Moughan, J. M. Wells, N. C. Roy, Live Faecalibacterium prausnitzii in an apical anaerobic model of the intestinal epithelial barrier. Cellular Microbiology 17, 226-240 (2015).

51. A. Pinnock, C. Murdoch, K. Moharamzadeh, S. Whawell, C. W. Douglas, Characterisation and optimisation of organotypic oral mucosal models to study Porphyromonas gingivalis invasion. Microbes Infect 16, 310-319 (2014).

52. W. Shin, A. Wu, M. W. Massidda, C. Foster, N. Thomas, D.-W. Lee, H. Koh, Y. Ju, J. Kim, H. J. Kim, A robust longitudinal co-culture of obligate anaerobic gut microbiome with human intestinal epithelium in an anoxic-oxic interface-on-a-chip. Frontiers in Bioengineering and Biotechnology 7, 13 (2019).

53. M. Marzorati, B. Vanhoecke, T. De Ryck, M. Sadaghian Sadabad, I. Pinheiro, S. Possemiers, P. Van den Abbeele, L. Derycke, M. Bracke, J. Pieters, T. Hennebel, H. J. Harmsen, W. Verstraete, T. Van de Wiele, The $\mathrm{HMI}^{\mathrm{TM}}$ module: a new tool to study the host-microbiota interaction in the human gastrointestinal tract in vitro. BMC Microbiol 14, 133 (2014).

54. H. J. Kim, D. Huh, G. Hamilton, D. E. Ingber, Human gut-on-a-chip inhabited by microbial flora that experiences intestinal peristalsis-like motions and flow. Lab Chip 12, 2165-2174 (2012).

55. H. J. Kim, H. Li, J. J. Collins, D. E. Ingber, Contributions of microbiome and mechanical deformation to intestinal bacterial overgrowth and inflammation in a human gut-on-a-chip. Proc Natl Acad Sci U S A 113, E7-15 (2016).

56. P. Shah, J. V. Fritz, E. Glaab, M. S. Desai, K. Greenhalgh, A. Frachet, M. Niegowska, M. Estes, C. Jäger, C. Seguin-Devaux, F. Zenhausern, P. Wilmes, A microfluidics-based in vitro model of the gastrointestinal human-microbe interface. Nat Commun 7, 11535 (2016).

57. V. V. Thacker, N. Dhar, K. Sharma, R. Barrile, K. Karalis, J. D. McKinney, A lung-on-chip infection model reveals protective and permissive roles of alveolar epithelial cells in tuberculosis. bioRxiv, 2020.02.03.931170 (2020).

58. G. G. Einarsson, D. M. Comer, L. Mcllreavey, J. Parkhill, M. Ennis, M. M. Tunney, J. S. Elborn, Community dynamics and the lower airway microbiota in stable chronic obstructive pulmonary disease, smokers and healthy non-smokers. Thorax 71, 795 (2016).

59. D. Papazian, T. Chhoden, M. Arge, T. Vorup-Jensen, C. H. Nielsen, K. Lund, P. A. Würtzen, S. Hansen, Effect of polarization on airway epithelial conditioning of monocyte-derived dendritic cells. Am J Respir Cell Mol Biol 53, 368-377 (2015). 
664

665

666

667

668

669

670

671

672

673

674

675

676

677

678

679

680

681

682

683

684

685

686

687

688

689

690

691

692

60. C. E. Stewart, E. E. Torr, N. H. Mohd Jamili, C. Bosquillon, I. Sayers, Evaluation of differentiated human bronchial epithelial cell culture systems for asthma research. J Allergy (Cairo) 2012, 943982 (2012).

61. C. I. Grainger, L. L. Greenwell, D. J. Lockley, G. P. Martin, B. Forbes, Culture of Calu-3 cells at the air interface provides a representative model of the airway epithelial barrier. Pharm Res 23, 1482-1490 (2006).

62. W. E. Finkbeiner, S. D. Carrier, C. E. Teresi, Reverse transcription-polymerase chain reaction (RTPCR) phenotypic analysis of cell cultures of human tracheal epithelium, tracheobronchial glands, and lung carcinomas. Am J Respir Cell Mol Biol 9, 547-556 (1993).

63. P. LeSimple, J. Goepp, M. L. Palmer, S. C. Fahrenkrug, S. M. O'Grady, P. Ferraro, R. Robert, J. W. Hanrahan, Cystic fibrosis transmembrane conductance regulator is expressed in mucin granules from Calu-3 and primary human airway epithelial cells. Am J Respir Cell Mol Biol 49, 511-516 (2013).

64. D. Worlitzsch, R. Tarran, M. Ulrich, U. Schwab, A. Cekici, K. C. Meyer, P. Birrer, G. Bellon, J. Berger, T. Weiss, K. Botzenhart, J. R. Yankaskas, S. Randell, R. C. Boucher, G. Döring, Effects of reduced mucus oxygen concentration in airway Pseudomonas infections of cystic fibrosis patients. J Clin Invest 109, 317-325 (2002).

65. P. K. Singh, A. L. Schaefer, M. R. Parsek, T. O. Moninger, M. J. Welsh, E. P. Greenberg, Quorumsensing signals indicate that cystic fibrosis lungs are infected with bacterial biofilms. Nature 407, 762764 (2000).

66. K. Martens, P. W. Hellings, B. Steelant, Calu-3 epithelial cells exhibit different immune and epithelial barrier responses from freshly isolated primary nasal epithelial cells in vitro. Clin Transl Allergy 8, 40 (2018).

67. Y. Liao, G. K. Smyth, W. Shi, The R package Rsubread is easier, faster, cheaper and better for alignment and quantification of RNA sequencing reads. Nucleic Acids Res 47, e47 (2019).

68. M. I. Love, W. Huber, S. Anders, Moderated estimation of fold change and dispersion for RNA-seq data with DESeq2. Genome Biol 15, 550 (2014).

69. M. Stephens, False discovery rates: a new deal. Biostatistics 18, 275-294 (2017). 
ACKNOWLEDGEMENTS

694 We thank the University of Minnesota Fabrication Shop for the custom design of our anaerobic chamber 695 culture manifold. Funding. This work was supported by the National Heart Lung and Blood Institute 696 (HL136919), a Cystic Fibrosis Postdoctoral Fellowship to P.J.M. (MOORE20F0), a National Institutes of 697 Health fellowship (\#T90DE0227232) awarded through the National Institute of Dental and Craniofacial 698 Research to S.K.L., and an American Society for Microbiology Undergraduate Research Fellowship to 699 L.A.K. Author contributions. P.J.M. and R.C.H. were responsible for study design and wrote the 700 manuscript. P.J.M. T.D.W., L.A.K., S.J.A., S.K.L., and R.C.H., collected and analyzed the data and edited 701 the manuscript. T.D.W., S.J.A., and S.K.L. processed and analyzed RNA sequencing data. S.M.O. 702 contributed cell lines and assisted with experiments. Competing interests. The authors declare they have 703 no competing interests. Data and materials availability. All data needed to evaluate the conclusions are 704 present in the paper, Supplementary Materials, or available online at https://github.com/Hunter-LabUMN/Moore_PJ_2020. 